\title{
Prognostic Significance and Predictive Factors of Lymph Node Metastasis in Resectable Intrahepatic Cholangiocarcinoma
}

\author{
Navarro J G, Rho SY, Lee J H, Choi GH*, Han DH, \\ Kim SK and Choi JS \\ Department of Surgery, Yonsei University College of \\ Medicine, Korea \\ *Corresponding author: Choi GH, Department of \\ Surgery, Yonsei University College of Medicine, Yonsei- \\ ro, Seodaemun-gu, Korea
}

Received: December 20, 2018; Accepted: February 08, 2019; Published: February 15, 2019

\begin{abstract}
Background: Lymph node metastasis portend a worse prognosis following resection of Intrahepatic Cholangiocarcinoma (ICC); however, the role of lymphadenectomy is still controversial and not routinely performed. In this study, we investigated the oncologic significance and predictive factors of lymph node metastasis in patients with ICC, which can potentially influence decision making for the patient's oncologic benefit with lymphadenectomy.
\end{abstract}

Materials and Methods: We retrospectively reviewed patients who underwent curative-intent surgery for intrahepatic cholangiocarcinoma between 2001 and 2014. The data was collected from the electronic medical record database of the hospital.

Results: A total of 168 patients were included in the study. Multivariable analysis revealed that: age $>65$ years, lymph node metastasis, tumor size $>5$ $\mathrm{cm}$ and periductal infiltrating tumor morphology, were independently associated with poor OS $(\mathrm{P}<0.05)$; moreover, tumor size $>5 \mathrm{~cm}$, periductal infiltrating tumor morphology, multiple tumor, vascular invasion, and lymph node metastasis, were independently associated with increased risk of tumor recurrence $(P<0.05)$. The CT finding of enlarged lymph nodes and CA 19-9 $>120 \mathrm{IU} / \mathrm{ml}$ were preoperative predictors for lymph node metastasis; however, the sensitivity and specificity were only $62.5 \%$ and $88.0 \%$, respectively, to identify lymph node metastasis.

Conclusion: Lymph node metastasis is associated with poor overall survival and disease-free survival following curative-intent resection in patients with ICC. Routine lymph node dissection for preoperatively diagnosed ICC should be recommended to properly assess the lymph node status of patients with ICC.

Keywords: Intrahepatic cholangiocarcinoma; Lymph node; Survival

\section{Abbreviations}

ICC: Intrahepatic Cholangiocarcinoma; CA19-9: Carbohydrate Antigen 19-9; CEA: Carcinoembryonic Antigen; CT: Computed Tomography; MRCP: Magnetic Resonance Imaging with Cholangiography; PET: Positron Emission Tomography; AJCC: American Joint Committee on Cancer; OS: Overall Survival; DFS: Disease-Free Survival

\section{Introduction}

Intrahepatic Cholangiocarcinoma (ICC) is the second most common primary liver malignancy [1]. In Korea, it represents approximately $10 \%$ of liver cancers $(8.8 \%$ for males and $10.6 \%$ for females) and this proportion increases by year with an estimated annual percentage change of $7.9 \%$ and $10.6 \%$ for males and females, respectively, from 1999 to 2005 [2]. Up to date, there is limited data regarding the effectiveness of adjuvant chemotherapy for ICC; therefore, surgical resection remains the only definitive treatment available [3]. Although survival has improved in the last decade, [4] the 5-year survival still ranges from $20-40 \%$, [5-7] and the recurrence rates remain as high as $50-60 \%[8,9]$ even after complete surgical resection.

As a result, it is important to recognize prognostic factors associated with long-term survival for patients with ICC for risk stratification after surgery. Most prominently, the lymph node status which can be influenced by the surgeon through an adequate lymphadenectomy. Several reports have documented the incidence of lymph node metastasis in patients with ICC as high a 45-62\% [10,11]. Moreover, although its oncologic significance is well-documented in different studies, [12] the role of lymphadenectomy is still controversial and not even routinely performed [13]. Therefore, we sought to investigate the prognostic factors of patients with ICC who underwent curative intent surgery. In addition, we sought to identify the preoperative determinants of lymph node metastasis in patients with ICCs as it might potentially influence the decision making and the patient's oncologic benefit on lymphadenectomy.

\section{Materials and Methods}

\section{Study design}

A retrospective review of all patients with a histologically confirmed intrahepatic cholagiocarcinoma who underwent curative 

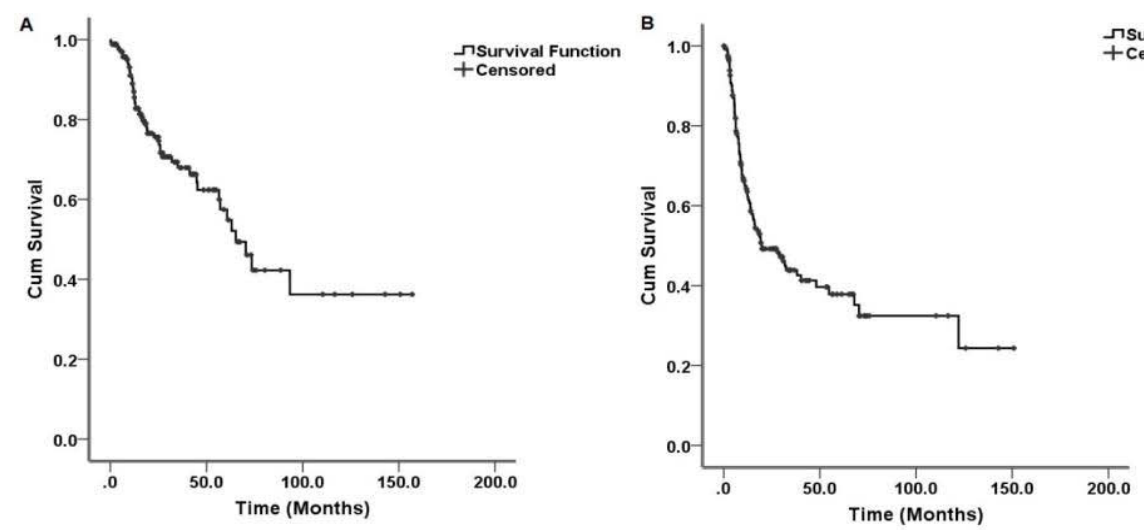

Figure 1: (A) Overall survival Kaplan-Mier curve of patients with ICC after curative-intent resection, (B) Disease-free survival.

Table 1: Clinipathologic characteristics of patients.

\begin{tabular}{|c|c|}
\hline Variables & $N=168$ \\
\hline \multicolumn{2}{|l|}{ Age } \\
\hline$<65$ & $87(51.8 \%)$ \\
\hline$\geq 65$ & $81(48.2 \%)$ \\
\hline \multicolumn{2}{|l|}{ Sex } \\
\hline Male & $97(57.7 \%)$ \\
\hline Female & $71(42.3 \%)$ \\
\hline \multicolumn{2}{|l|}{ CA $19-9 \mathrm{IU} / \mathrm{ml}$} \\
\hline$<72$ & $75(44.6)$ \\
\hline$\geq 72$ & $70(41.7)$ \\
\hline \multicolumn{2}{|l|}{ Tumor size $(\mathrm{cm})$} \\
\hline$\leq 5$ & $80(47.6 \%)$ \\
\hline$>5$ & $88(52.4 \%)$ \\
\hline \multicolumn{2}{|l|}{ Number of lesions } \\
\hline Solitary & $137(81.5 \%)$ \\
\hline Multiple & $31(18.5 \%)$ \\
\hline Biliary invasion & $92(54.8 \%)$ \\
\hline \multicolumn{2}{|l|}{ Perineural invasion } \\
\hline Vascular invasion & $105(62.5 \%)$ \\
\hline \multicolumn{2}{|l|}{ Tumor Grade } \\
\hline Well/Moderately differentiated & $129(76.8 \%)$ \\
\hline Poorly/Undifferentiated & $29(12.7 \%)$ \\
\hline \multicolumn{2}{|c|}{ Metastatic lymph nodes (AJCC $8^{\text {th }}$ ed., pN) } \\
\hline Nx & $29(17.3 \%)$ \\
\hline NO & $96(57.1 \%)$ \\
\hline N1 & $43(25.6 \%)$ \\
\hline \multicolumn{2}{|l|}{ Tumor Morphology } \\
\hline Mass forming & $115(68.5 \%)$ \\
\hline Periductal infiltrating & $14(8.3)$ \\
\hline Intraductal growth & $21(12.5 \%)$ \\
\hline Mixed type & $17(10.1 \%)$ \\
\hline Positive resection margin (R1) & $24(14.3 \%)$ \\
\hline
\end{tabular}

\begin{tabular}{|l|c|}
\hline TNM stage (AJCC $8^{\text {th }}$ edition) & $11(6.5 \%)$ \\
\hline IA & $15(8.9 \%)$ \\
\hline IB & $8(4.8 \%)$ \\
\hline II & $62(36.9 \%)$ \\
\hline IIIB & $43(25.6 \%)$ \\
\hline Unknown & $29(17.3 \%)$ \\
\hline Surgical resection & \\
\hline Less than hemihepatectomy & $35(20.8 \% 0$ \\
\hline Right hemihepatectomy & $56(33.3 \%)$ \\
\hline Left hepatectomy & $57(33.9 \%)$ \\
\hline Central bisectionectomy & $4(2.4 \%)$ \\
\hline Extended hemihepatectomy & $15(8.9 \%)$ \\
\hline Lymph node dissection (LND) & $29(17.3 \%)$ \\
\hline No LND & $139(82.7 \%)$ \\
\hline Standard LND & \\
\hline CA: Cancer Antigen: AJCC American Jont Com & \\
\hline
\end{tabular}

CA: Cancer Antigen; AJCC: American Joint Committee on Cancer; LND: Lymph Node Dissection

intent resection in Severance hospital, Yonsei University College of Medicine, Seoul, Korea, between 2000 and 2014. Patients with incomplete clinical and histologic data were excluded from this study. Also excluded were patients with other underlying malignancies. The study protocol was approved by the Yonsei Institutional Review Board.

\section{Clinicopathologic data}

The data was collected from the electronic medical record database of the hospital. Patients' demographic characteristics, clinical presentations, preoperative comorbidities, perioperative outcomes, imaging studies, and laboratory results were reviewed and analyzed. Preoperative evaluation including: carbohydrate antigen 19-9 (CA199), Computed Tomography (CT), Magnetic Resonance Imaging with Cholangiography (MRCP) and Positron Emission Tomography (PET), were reviewed. Tumor characteristics such as tumor size, type, number, and the presence or absence of enlarged lymph nodes were obtained from the report of preoperative imaging studies. An enlarged lymph nodes on preoperative imaging was determined as exceeding 
Table 2: Clinicopathologic factors associated with overall survival.

\begin{tabular}{|c|c|c|c|c|c|c|}
\hline \multirow{2}{*}{ Clinicopathologic factors } & \multirow{2}{*}{$\mathrm{N}$} & \multicolumn{2}{|c|}{ Univariable } & \multicolumn{3}{|c|}{ Multivariable } \\
\hline & & Means (Months) & $P$ value & $P$ value & $\mathrm{HR}$ & $95 \% \mathrm{Cl}$ \\
\hline \multicolumn{7}{|l|}{ Age (years) } \\
\hline$<65$ & 87 & 105.6 & & & & \\
\hline$\geq 65$ & 81 & 56.1 & 0.0001 & 0.006 & 2.31 & $1.264-4.210$ \\
\hline \multicolumn{7}{|l|}{ Sex } \\
\hline Male & 97 & 78.2 & & & & \\
\hline Female & 71 & 86 & 0.621 & & & \\
\hline \multicolumn{7}{|l|}{ CA 19-9 (U/mL) } \\
\hline$<72$ & 75 & 84.2 & & & & \\
\hline$>72$ & 70 & 65.6 & 0.039 & 0.345 & 1.34 & $0.730-2.460$ \\
\hline \multicolumn{7}{|l|}{ Tumor size $(\mathrm{cm})$} \\
\hline$\leq 5$ & 80 & 94.5 & & & & \\
\hline$>5$ & 88 & 68.3 & 0.007 & 0.014 & 2.33 & $1.186-4.575$ \\
\hline Number of lesions & 137 & 84.5 & & & & \\
\hline Solitary & 31 & 51.3 & 0.186 & & & \\
\hline \multicolumn{7}{|l|}{ Multiple } \\
\hline \multicolumn{7}{|l|}{ Bile duct invasion } \\
\hline No & 92 & 97.9 & & & & \\
\hline Yes & 76 & 52 & 0.027 & 0.458 & 1.27 & $0.677-2.376$ \\
\hline \multicolumn{7}{|l|}{ Vascular invasion } \\
\hline No & 63 & 107.6 & & & & \\
\hline Yes & 105 & 62.6 & 0.0001 & 0.093 & 2.01 & $0.891-4.523$ \\
\hline Yes & 65 & 58.5 & 0.001 & 0.838 & 1.08 & $0.536-2.158$ \\
\hline \multicolumn{7}{|l|}{ Metastatic lymph } \\
\hline No & 96 & 99.9 & 0.001 & 0.023 & 2.22 & $1.115-4.427$ \\
\hline Yes & 43 & 30.5 & & & & \\
\hline Tumor morphology & & & 0.002 & 0.006 & & \\
\hline Intraductal growth & 21 & 110.9 & Ref & Ref & & \\
\hline Mass forming & 115 & 90.6 & 0.141 & 0.828 & & \\
\hline Periductal infiltrating & 14 & 28.6 & 0.002 & 0.006 & 3.96 & $1.496-10.486$ \\
\hline Mixed type & 17 & 48.9 & 0.025 & 0.758 & & \\
\hline \multicolumn{7}{|l|}{ Tumor Grades } \\
\hline Well/Moderately differentiated & 129 & 82.1 & & & & \\
\hline Poorly/Undifferentiated & 29 & 50.5 & 0.151 & & & \\
\hline \multicolumn{7}{|l|}{ Resection margin } \\
\hline Ro & 144 & 80.5 & & & & \\
\hline R1 & 24 & 70.9 & 0.443 & & & \\
\hline
\end{tabular}

CA: Cancer Antigen; Cl: Confidence Interval; HR: Hazard Ratio; AJCC: American Joint Committee on Cancer

the short axis diameter of $8 \mathrm{~mm}$ in the gastrohepatic ligament, $7 \mathrm{~mm}$ in the porta hepatis, $10 \mathrm{~mm}$ in the portocaval space, and $9 \mathrm{~mm}$ in the paraaortic region [14]. Enlarged lymph nodes on preoperative imaging were defined as clinical N1 whereas clinical N0 if no enlarged lymph nodes. The standard lymph node dissection in this cohort was defined as removal of the hilar and hepatoduodenal ligament (station 12) or more such as the retropancreatic, the common hepatic artery, the celiac axis, the left gastric artery, or the caval lymph nodes.

Pathology review was performed by an experienced hepatobiliary pathologist. Tumor growth type, grade, vascular invasion, 
Table 3: Clinicopathologic factors associated with disease-free survival.

\begin{tabular}{|c|c|c|c|c|c|c|}
\hline \multirow{2}{*}{ Clinicopathologic factors } & \multirow{2}{*}{$\mathrm{N}$} & \multicolumn{2}{|c|}{ Univariable } & \multicolumn{3}{|c|}{ Multivariable } \\
\hline & & Means (Months) & $P$ value & $P$ value & $\mathrm{HR}$ & $95 \% \mathrm{Cl}$ \\
\hline \multicolumn{7}{|l|}{ Age } \\
\hline$<65$ & 87 & 56 & & & & \\
\hline$\geq 65$ & 81 & 64.6 & 0.965 & & & \\
\hline \multicolumn{7}{|l|}{ Sex } \\
\hline Male & 97 & 51.6 & 0.405 & & & \\
\hline Female & 71 & 66.2 & & & & \\
\hline \multicolumn{7}{|l|}{ CA 19-9 (U/mL) } \\
\hline$<72$ & 75 & 67.8 & 0.031 & 0.37 & 1.28 & $0.749-2.172$ \\
\hline$\geq 72$ & 70 & 48.7 & & & & \\
\hline$\leq 5$ & 80 & 71.2 & 0.0001 & 0.013 & 2.14 & $1.176-3.884$ \\
\hline$>5$ & 88 & 38.8 & & & & \\
\hline \multicolumn{7}{|l|}{ Number of lesions } \\
\hline Solitary & 137 & 66.2 & 0.001 & 0.007 & 2.26 & $1.254-4.053$ \\
\hline Multiple & 31 & 21.8 & & & & \\
\hline \multicolumn{7}{|l|}{ Bile duct invasion } \\
\hline No & 92 & 67.9 & 0.116 & & & \\
\hline Yes & 76 & 32.1 & & & & \\
\hline \multicolumn{7}{|l|}{ Vascular invasion } \\
\hline No & 63 & 88.8 & 0.0001 & 0.012 & 2.31 & $1.201-4.431$ \\
\hline Yes & 105 & 37.8 & & & & \\
\hline Yes & 65 & 37.3 & & & & \\
\hline \multicolumn{7}{|l|}{ Metastatic lymph node } \\
\hline No & 96 & 61.2 & 0.0001 & 0.006 & 2.15 & $1.247-3.688$ \\
\hline Yes & 43 & 18.2 & & & & \\
\hline \multicolumn{7}{|l|}{ Tumor morphology } \\
\hline Intraductal growth & 21 & 97.2 & Ref & Ref & & \\
\hline Mass forming & 115 & 57.8 & 0.002 & 0.243 & 0.47 & $0.132-1.668$ \\
\hline Periductal infiltrating & 14 & 12 & 0.0001 & 0.0001 & 4.68 & $2.122-10.318$ \\
\hline Mixed type & 17 & 40.7 & 0.014 & 0.22 & 0.61 & $0.275-1.346$ \\
\hline \multicolumn{7}{|l|}{ Tumor Grades } \\
\hline Well/Moderately differentiated & 129 & 69.4 & 0.0001 & 0.198 & 1.48 & $0.813-2.710$ \\
\hline Poorly/Undifferentiated & 29 & 17 & & & & \\
\hline \multicolumn{7}{|l|}{ Resection margin } \\
\hline Ro & 144 & 59.9 & 0.861 & & & \\
\hline $\mathrm{R} 1$ & 24 & 50.9 & & & & \\
\hline
\end{tabular}

CA: Cancer Antigen, Cl: Confidence Interval; HR: Hazard Ratio; AJCC: American Joint Committee on Cancer

multicentricity, and lymph node status were obtained. The tumor growth pattern was classified as being of mass-forming type, periductal infiltrating type, intraductal, and mixed type (massforming/periductal infiltrating type) according to the Liver Cancer Study Group of Japan [15]. The pathological stage was defined according to the $8^{\text {th }}$ edition of the American Joint Committee on Cancer $8^{\text {th }}$ edition (AJCC) [16].

\section{Study outcomes}

The primary endpoints of this study were the long-term survival outcomes of patients with intrahepatic cholangiocarcinoma after 
curative-intent resection. Overall survival was calculated from the date of surgery to the date of death or last follow-up. Disease-free survival was calculated from the date of surgery to the date of recurrence or last follow-up. Patients were also followed up regularly for serum CA 19-9, Carcinoembryonic Antigen (CEA) level determination, and computed tomography scan to detect tumor recurrence.

\section{Statistical analysis}

Statistical analysis was performed using IBM SPSS Statistics, version 22 (SPSS Inc., Chicago, IL, USA). Categorical variables were expressed as frequencies (\%), whereas continuous variables were presented as means with their range or \pm standard deviation. The means of continuous variables were compared using an independent sample t-test. Categorical variables were compared using the Pearson $P^{2}$ test. The Receiver Operating Characteristic (ROC) curve analysis was used to determine the optimal cutoff value of CA 19-9 in determining lymph node metastasis. Overall survival and diseasefree survival were estimated using the Kaplan-Meier method and compared using a log-rank test. A multivariable Cox proportional hazards model was applied to identify the statistically significant independent prognostic factors for overall survival and disease-free survival. Association between the preoperative variables and lymph nodes metastases was tested, as continues and categorical, using logistic regression analysis. A P-value of $<.05$ was used to define statistical significance.

\section{Results}

\section{Clinicopathologic characteristics}

A total of 179 patients who underwent curative liver resection for ICC were initially identified. Among them, only 168 patients met the inclusion criteria. The median age was 64 years (range, $360-84$ years). The characteristics of patients are summarized in (Table 1). With a median follow-up period of 21 months (range, 1-156 months), 88 patients (52\%) had a recurrence and 53 patients (32\%) died in this cohort. Moreover, among 88 patients with tumor recurrence, 77 (86.5\%) recurred within 2 years of follow-up.

\section{Factors associated with Overall Survival (OS)}

The cumulative 1-year, 3-year, and 5-year OS rates were $86.9 \%$, $67.9 \%$, and $54.9 \%$, respectively (Figure $1 \mathrm{~A}$ ). The median OS was 65 months. Factors associated with poor OS included age $>65$ years, CA19-9 >72 U/ml, vascular invasion, perineural invasion, bile duct invasion, tumor size $>5 \mathrm{~cm}$, periductal infiltrating tumor morphology, and metastatic lymph nodes ( $\mathrm{pN} 1)(\mathrm{P}<0.05)$. On multivariable analysis, Age $>65$ years (HR, 2.307; 95\% CI, 1.264-4.210; $\mathrm{P}=0.006$ ), lymph node metastasis ( $\mathrm{HR}, 2.222 ; 95 \% \mathrm{CI}, 1.115-4.427 ; \mathrm{P}=0.023$ ), tumor size $>5 \mathrm{~cm}(\mathrm{HR}, 2.330 ; 95 \% \mathrm{CI}, 1.186-4.575 ; \mathrm{P}=.014)$, and periductal infiltrating tumor morphology (HR, 3.961; 95\% CI, 1.49610.486; $\mathrm{P}=0.006$ ) were independently associated with poor OS. Table 2 detailed the clinicopathologic factors associated with OS.

\section{Factors associated with Disease-Free Survival (DFS)}

The median DFS was 19.7 months, and the cumulative 1-year, 3-year, 5-year DFS rates were 63.5\%, 43.8\%, 37.8\%, respectively (Table 3 \& Figure 1B). Factors associated with increased risk of recurrence included CA19-9 $>72 \mathrm{IU} / \mathrm{ml}$, multiple tumor, poorly/undifferentiated tumor, vascular invasion, perineural invasion, tumor size $>5 \mathrm{~cm}$, periductal infiltrating tumor morphology, and lymph node metastasis
$(\mathrm{P}<0.05)$. On multivariable analysis, tumor size $>5 \mathrm{~cm}(\mathrm{HR}, 2.137 ; 95 \%$ $\mathrm{CI}, 1.176-3.884 ; \mathrm{P}=0.013)$, periductal infiltrating tumor morphology (HR, 4.679; 95\% CI, 1.247-10.318; $\mathrm{P}=0.0001$ ), multiple tumor (HR, 2.255; 95\% CI, 1.254-4.070); $\mathrm{P}=0.007$ ), vascular invasion (HR, 2.307; 95\% CI, 1.201-4.431); $\mathrm{P}=0.012$ ) and lymph node metastasis (HR, 2.145; 95\% CI, 1.247-3.688; $\mathrm{P}=0.006$ ) were independently associated with increased risk of tumor recurrence.

\section{Preoperative factors associated with lymph node metastasis}

Among 139 patients who underwent standard lymph node dissection, 42 (30.2\%) had positive lymph node metastasis on final histopathology report. To identify the preoperative predictive factors for lymph node metastasis in patients with ICC, we compared the preoperative variables among patient with lymph node metastasis and no lymph node metastasis by using logistic regression analysis. We included age $>65$ years, gender, CA $19-9$, and preoperative CT scan tumor size $>5 \mathrm{~cm}$, tumor morphology (mass forming, periductal infiltrating, and intraductal growth) and clinically enlarged lymph node on CT scan. A Receiver Operating Characteristic (ROC) curve analysis was used to determine the optimal cutoff value of CA 19-9 to predict pathologic lymph node. The optimal cutoff value was $120 \mathrm{IU} /$ $\mathrm{ml}$ (sensitivity, 55.3\%; and specificity, 70.2\%) with an Area under the Curve (AOC) 0.619 (95\% CI, 0.505-0.733, $\mathrm{P}=0.035$ ).

The CT findings of enlarged lymph nodes (clinical N1) and CA $19-9>120 \mathrm{IU} / \mathrm{ml}$ were predictive of $\mathrm{pN} 1$ in univariable analysis, and remained independent predictors in multivariable analysis (Table 4). Furthermore, we identified 16 patients with $\mathrm{cN} 1$ and CA 19-9 >120 $\mathrm{IU} / \mathrm{ml}$ (high risk group), 28 patients with $\mathrm{cN} 0$ and CA 19-9 >120 IU/ $\mathrm{ml}$ (intermediate risk group), 20 patients with $\mathrm{cN} 1$ and CA $19-9<120$ $\mathrm{IU} / \mathrm{ml}$ (intermediate risk group), and 50 patients cN0 and CA 19-9 $<170 \mathrm{IU} / \mathrm{ml}$ (low risk group). Table 5 summarizes the incidence of lymph node metastasis according to the CA 19-9 and clinical lymph node status. Notably, the high-risk group is associated with $62.5 \%$ risk of lymph node metastasis following curative resection compared to $30-35 \%$ in the intermediate risk groups and $12.0 \%$ in the low-risk group $(\mathrm{P}=0.001)$; however, the sensitivity was only $62.5 \%$ for the high risk group to identify lymph node metastasis and specificity $88.0 \%$ for the low risk group to rule out lymph node metastasis.

\section{Discussion}

The standard of care for intrahepatic cholangiocarcinoma remains complete surgical resection. However, due to the rarity of the disease, most of the reports on the management of ICC are from small retrospective reviews of data from individual institutions; therefore, intrahepatic cholangiocarcinoma after surgery is still associated with poor prognosis. Moreover, the reported 5-year overall survival of ICC after complete surgical resection in studies ranges from 20$40 \%$ [5-7,17]. Our present study however, had demonstrated a high 5 -year survival rate of $54.9 \%$, slightly higher than reported in studies. However, despite our aggressive surgical approach for patients with ICC, we were able to achieve $85.6 \% \mathrm{R} 0$ resection rate but the 5 -year DFS remains low (37.8\%). Notably, although R1 resection was not associated with poor survival outcome in this cohort, several reports had demonstrated a poor DFS and OS with R1 resection [18-20].

Our present cohort has identified various clinicopathologic 
Table 4: Logistic regression analysis of factors predictive for pathologic lymph node metastasis.

Table 5: Incidence of lymph node metastasis according to the presence or absence of risk factors.

\begin{tabular}{|l|c|c|}
\hline Risk factors & Pathologic N0 (\%) & Pathologic N1 (\%) \\
\hline High risk & & \\
\hline Clinical N0 + CA $19-9>120 \mathrm{IU}$ & $6(37.5 \%)$ & $10(62.5 \%)$ \\
\hline Intermediate risk & & $6(30 \%)$ \\
\hline Clinical N1 + CA $19-9<120 \mathrm{IU} / \mathrm{ml}$ & $14(70 \%)$ & $10(35.7 \%)$ \\
\hline Clinical N0 + CA $19-9>120 \mathrm{IU} / \mathrm{ml}$ & $18(64.3 \%)$ & $6(12.0 \%)$ \\
\hline Low risk & & \\
\hline Clinical N0 + CA $19-9<120 \mathrm{IU} / \mathrm{ml}$ & $44(88.0 \%)$ & \\
\hline Sensitivity of High risk: $62.5 \%$ & & \\
\hline Specificity of low risk: $88.0 \%$ & & \\
\hline Positive predictive value: 62.5 & & \\
\hline Negative predictive value: $88.0 \%$ & & \\
\hline Accuracy: $75.2 \%$ & & \\
\hline
\end{tabular}

cN0: no lymph node in CT scan (clinical N0); cN1: Presence of lymph node on CT scan (clinical N1); CA: Cancer Antigen

factors associated with poor long-term survival after curative-intent surgery. It is important to identify these prognostic factors for patients' risk stratification, close follow-up monitoring, and future planning of adjuvant therapy. We have found that independent predictors of OS were age $>65$ years, the presence of lymph node metastases, tumor size $>5 \mathrm{~cm}$, and periductal infiltrating growth type tumor. Moreover, independent factors associated with decrease DFS were tumor size $>5 \mathrm{~cm}$, periductal infiltrating growth type tumor, multiple tumor, vascular invasion, and lymph node metastasis. These factors, however, had been demonstrated as important determinant of OS and DFS in different studies [5,6,17,21-23].

Frequently cited as important prognostic factors for overall survival for patients with intrahepatic cholagiocarcinoma are the presence of vascular invasion and multiple tumor lesions [24,25]. AJCC $7^{\text {th }}$ and $8^{\text {th }}$ edition emphasizes the clinical importance of these two factors in the $\mathrm{T}$ stage classification for intrahepatic cholangiocarcinoma. On the contrary, multiple tumors were not associated with poor overall survival in this cohort; furthermore, although it was proven to be associated with poor overall survival in the univariate analysis, vascular invasion was not found to be an independent factor associated with overall survival. Nevertheless, both these factors were found to be an independent variable for decreased disease-free survival after curative-intent resection. These findings are the same as Weber et al. [26]. reported that together with tumor size, vascular invasion, and multiple lesions were associated with increased risk of tumor recurrence after curative-intent resection.

Moreover, tumor size of $>5 \mathrm{~cm}$ and the periductal infiltrating type of tumor is found to be associated with poor overall survival, as well disease-free survival in this present cohort. Tumor size was found to be associated with poor pathologic outcome such as vascular invasion and poor tumor differentiation [27] and hence associated with poor prognosis $[28,29]$. Some studies however, had shown that tumor size was not a significant predictor of overall survival [30,31]. Periductal infiltrating tumor is also a well-recognized prognostic factor associated with poor long-term survival $[15,32]$. In AJCC $7^{\text {th }}$ edition, it was used to identify the $\mathrm{T}$ classification into $\mathrm{T} 4$, however, it was no longer part of the T classification in the AJCC $8^{\text {th }}$ edition.

Furthermore, in this present cohort, lymph node metastasis portends a poor long-term survival. This finding was consistently reported in various studies [29,33]. As such, in order to properly determine the prognosis of patient with intrahepatic cholangiocarcinoma, a thorough evaluation of the lymph node status by adequate lymph node dissection must be emphasized. Several reports had documented as high as 45-62\% incidence of lymph node metastasis in patients with ICC $[10,11]$ and its oncologic significance was also well-documented in different studies [34]. However, until recently, not all patients with ICC underwent lymph node dissection [10]. Thus, in our cohort, we tried to identify those preoperative determinants of lymph node metastasis in patients with ICCs as it might potentially influence the decision making about who might benefit from lymphadenectomy. Our result had demonstrated that patient with clinically lymph node metastasis $(\mathrm{cN} 1)$ on CT scan and elevated CA 19-9>120 IU/ml significantly associated with pathologic lymph node metastasis in univariable and multivariable analysis. We classify these patients as high risk and has a $65.2 \%$ risks of lymph node metastases. Patients with either one of these 2 risk factors were classified as intermediate risk with $30-36 \%$ risk of lymph node metastasis whereas neither of these 2 risk factors was associated with $12 \%$ risk of lymph node metastasis. The same observation reported by Nanashima et al. [35] that the combination of positive CT findings and high CA19-9 level had the highest positive rate for node metastasis for biliary and pancreatic carcinoma. Although CT scan has a low accuracy in detecting lymph node metastasis in patients with cholangiocarcinoma, [36-38] CA 19-9 was notably associated with advanced disease and lymph node metastasis in some studies $[39,40]$. However, given that this model has a sensitivity of only $62.5 \%$ and more importantly a specificity of only $88.0 \%$, we still advocate a routine lymph node dissection considering that $12 \%$ of patient 
categorized as low risk may harbor an occult lymph node metastasis. As such, up to date, there is still no valuable tools with high sensitivity and specificity to identify preoperatively the lymph node metastasis for intrahepatic cholangiocarcinoma.

This study had several limitations. First, the selection bias that influenced the choice of treatment among our patients. For instance, patients with clinically small size tumor and clinically no lymph node on CT scan (cN0) were possibly selected to undergo liver resection only which might undoubtedly affect the survival outcome. Second, it is a single institution with a relatively small sample size. However, given the rarity of the ICC, this single institution study is one of the largest series that was reported. Nonetheless, our result should be further validated in a multicenter or randomized prospective study.

In summary, lymph node metastasis is associated with poor overall survival and disease-free survival in patients with ICC after curative-intent resection. A combination of preoperative CT scan and CA 19-9 for predicting lymph node metastasis has a sensitivity and specificity of $62.5 \%$ and $88.0 \%$ respectively. As such, routine lymph node dissection for preoperatively diagnosed intrahepatic cholangiocarcinoma should be recommended to properly assess the lymph node status of patients with intrahepatic cholangiocarcinoma.

\section{References}

1. Khan SA, Toledano MB, Taylor-Robinson SD. Epidemiology, risk factors, and pathogenesis of cholangiocarcinoma. HPB (Oxford). 2008; 10: 77-82.

2. Shin HR, Oh JK, Lim MK, Shin A, Kong HJ, Jung KW, et al. Descriptive Epidemiology of Cholangiocarcinoma and Clonorchiasis in Korea. J Korean Med Sci. 2010; 25: 1011-1016.

3. Weber SM, Ribero D, O'Reilly EM, Kokudo N, Miyazaki M, Pawlik TM Intrahepatic Cholangiocarcinoma: expert consensus statement. HPB. 2015; 17: $669-680$

4. Nathan H, Pawlik TM, Wolfgang CL, Choti MA, Cameron JL, Schulick RD. Trends in Survival after Surgery for Cholangiocarcinoma: A 30-Year Population-Based SEER Database Analysis. J Gastrointest Surg. 2007; 11: 1488-1497.

5. Cho SY, Park SJ, Kim SH, Han SS, Kim YK, Lee KW, et al. Survival analysis of intrahepatic cholangiocarcinoma after resection. Ann Surg Oncol. 2010; 17: $1823-1830$

6. Hyder O, Marques H, Pulitano C, Marsh JW, Alexandrescu S, Bauer TW, et al. A nomogram to predict long-term survival after resection for intrahepatic cholangiocarcinoma: an Eastern and Western experience. JAMA Surg. 2014; 149: $432-438$.

7. Konstadoulakis MM, Roayaie S, Gomatos IP, Labow D, Fiel MI, Miller CM, et al. Fifteen-year, single-center experience with the surgical management of intrahepatic cholangiocarcinoma: operative results and long-term outcome. Surgery. 2008; 143: 366-374.

8. Doussot A, Gonen M, Wiggers JK, Groot-Koerkamp B, DeMatteo RP, Fuks $D$, et al. Recurrence Patterns and Disease-Free Survival after Resection of Intrahepatic Cholangiocarcinoma: Preoperative and Postoperative Prognostic Models. J Am Coll Surg. 2016; 223: 493-505.

9. Hyder O, Hatzaras I, Sotiropoulos GC, Paul A, Alexandrescu S, Marques $\mathrm{H}$, et al. Recurrence after operative management of intrahepatic cholangiocarcinoma. Surgery. 2013; 153: 811-818.

10. Amini N, Ejaz A, Spolverato G, Maithel SK, Kim Y, Pawlik TM. Management of lymph nodes during resection of hepatocellular carcinoma and intrahepatic cholangiocarcinoma: a systematic review. J Gastrointest Surg. 2014; 18: 2136-2148.

11. Tsuji T, Hiraoka T, Kanemitsu K, Takamori H, Tanabe D, Tashiro S. Lymphatic spreading pattern of intrahepatic cholangiocarcinoma. Surgery.
2001; 129: 401-407.

12. Guglielmi A, Ruzzenente A, Campagnaro T, Valdegamberi A, Bagante $F$, Bertuzzo F, et al. Patterns and Prognostic Significance of Lymph Node Dissection for Surgical Treatment of Perihilar and Intrahepatic Cholangiocarcinoma. J Gastrointest Surg. 2013; 17: 1917-1928.

13. Adachi T, Eguchi S. Lymph node dissection for intrahepatic cholangiocarcinoma: a critical review of the literature to date. J Hepatobiliary Pancreat Sci. 2014; 21: 162-168.

14. Dorfman RE, Alpern MB, Gross BH, Sandler MA. Upper abdominal lymph nodes: criteria for normal size determined with CT. Radiology. 1991; 180: 319-322.

15. Sakamoto Y, Kokudo N, Matsuyama Y, Sakamoto M, Izumi N, Kadoya M, et al. Proposal of a new staging system for intrahepatic cholangiocarcinoma: Analysis of surgical patients from a nationwide survey of the Liver Cancer Study Group of Japan. Cancer. 2016; 122: 61-70.

16. Amin MB, Edge S, Greene F, Byrd DR, Brookland RK, Washington MK, et al. AJCC Cancer Staging Manual. 8 ed: Springer International Publishing; 2017.

17. Choi SB, Kim KS, Choi JY, Park SW, Choi JS, Lee WJ, et al. The Prognosis and Survival Outcome of Intrahepatic Cholangiocarcinoma Following Surgical Resection: Association of Lymph Node Metastasis and Lymph Node Dissection with Survival. Ann Surg Oncol. 2009; 16: 3048.

18. DeOliveira ML, Cunningham SC, Cameron JL, Kamangar F, Winter JM, Lillemoe KD, et al. Cholangiocarcinoma: Thirty-one-Year Experience With 564 Patients at a Single Institution. Ann Surg. 2007; 245: 755-762.

19. Spolverato G, Yakoob MY, Kim Y, Alexandrescu S, Marques HP, Lamelas J, et al. The Impact of Surgical Margin Status on Long-Term Outcome After Resection for Intrahepatic Cholangiocarcinoma. Ann Surg Oncol. 2015; 22: 4020-4028.

20. Yeh CN, Hsieh FJ, Chiang KC, Chen JS, Yeh TS, Jan YY, et al. Clinical effect of a positive surgical margin after hepatectomy on survival of patients with intrahepatic cholangiocarcinoma. Drug Des Devel Ther. 2015; 9: 163-174.

21. Chang ME, Lei HJ, Chen MH, Yeh YC, Li CP, Hung YP, et al. Evaluation of prognostic factors and implication of lymph node dissection in intrahepatic cholangiocarcinoma: 10-year experience at a tertiary referral center. J Chin Med Assoc. 2017; 80: 140-146.

22. Guglielmi A, Ruzzenente A, Campagnaro T, Pachera S, Valdegamberi A, Nicoli $P$, et al. Intrahepatic cholangiocarcinoma: prognostic factors after surgical resection. World J Surg. 2009; 33: 1247-1254.

23. Mavros MN, Economopoulos KP, Alexiou VG, Pawlik TM. Treatment and Prognosis for Patients with Intrahepatic Cholangiocarcinoma: Systematic Review and Meta-analysis. JAMA Surg. 2014; 149: 565-574.

24. Ohtsuka M, Ito $H$, Kimura F, Shimizu H, Togawa A, Yoshidome $H$, et al. Results of surgical treatment for intrahepatic cholangiocarcinoma and clinicopathological factors influencing survival. Br J Surg. 2002; 89: 15251531.

25. Okabayashi T, Yamamoto J, Kosuge T, Shimada K, Yamasaki S, Takayama $T$, et al. A new staging system for mass-forming intrahepatic cholangiocarcinoma: analysis of preoperative and postoperative variables. Cancer. 2001; 92: 2374-2383.

26. Weber SM, Jarnagin WR, Klimstra D, DeMatteo RP, Fong Y, Blumgart LH. Intrahepatic cholangiocarcinoma: resectability, recurrence pattern, and outcomes. J Am Coll Surg. 2001; 193: 384-3891.

27. Spolverato G, Ejaz A, Kim Y, Sotiropoulos GC, Pau A, Alexandrescu S, et al. Tumor size predicts vascular invasion and histologic grade among patients undergoing resection of intrahepatic cholangiocarcinoma. J Gastrointest Surg. 2014; 18: 1284-1291.

28. Morimoto Y, Tanaka Y, Ito T, Nakahara M, Nakaba H, Nishida T, et al. Longterm survival and prognostic factors in the surgical treatment for intrahepatic cholangiocarcinoma. Journal of Hepato-Biliary-Pancreatic Surgery. 2003; 10: 432-440.

29. Shen WF, Zhong W, Xu F, Kan T, Geng L, Xie F, et al. Clinicopathological 
and prognostic analysis of 429 patients with intrahepatic cholangiocarcinoma. World J Gastroenterol. 2009; 15: 5976-5982.

30. Choi SB, Kim KS, Choi JY, Park SW, Choi JS, Lee WJ, et al. The prognosis and survival outcome of intrahepatic cholangiocarcinoma following surgical resection: association of lymph node metastasis and lymph node dissection with survival. Ann Surg Oncol. 2009; 16: 3048-3056.

31. de Jong MC, Nathan H, Sotiropoulos GC, Paul A, Alexandrescu S, Marques $\mathrm{H}$, et al. Intrahepatic cholangiocarcinoma: an international multi-institutional analysis of prognostic factors and lymph node assessment. J Clin Oncol. 2011; 29: 3140-3145.

32. Shimada K, Sano T, Sakamoto Y, Esaki M, Kosuge T, Ojima H. Surgical outcomes of the mass-forming plus periductal infiltrating types of intrahepatic cholangiocarcinoma: a comparative study with the typical mass-forming type of intrahepatic cholangiocarcinoma. World J Surg. 2007; 31: 2016-2022.

33. Nakagawa T, Kamiyama T, Kurauchi N, Matsushita M, Nakanishi K, Kamach $\mathrm{H}$, et al. Number of lymph node metastases is a significant prognostic factor in intrahepatic cholangiocarcinoma. World J Surg. 2005; 29: 728-733.

34. Guglielmi A, Ruzzenente A, Campagnaro T, Valdegamberi A, Bagante $F$ Bertuzzo $F$, et al. Patterns and prognostic significance of lymph node dissection for surgical treatment of perihilar and intrahepatic cholangiocarcinoma. J Gastrointest Surg. 2013; 17: 1917-1928.
35. Nanashima A, Sakamoto I, Hayashi T, Tobinaga S, Araki M, Kunizaki M, et al. Preoperative Diagnosis of Lymph Node Metastasis in Biliary and Pancreatic Carcinomas: Evaluation of the Combination of Multi-detector CT and Serum CA19-9 Level. Dig Dis Sci. 2010; 55: 3617-3126.

36. Watadani T, Akahane M, Yoshikawa T, Ohtomo K. Preoperative assessment of hilar cholangiocarcinoma using multidetector-row CT: correlation with histopathological findings. Radiat Med. 2008; 26: 402-407.

37. Kim HJ, Lee DH, Lim JW, Ko YT. Multidetector computed tomography in the preoperative workup of hilar cholangiocarcinoma. Acta Radiologica. 2009; 50: 845-853.

38. Adachi T, Eguchi S, Beppu T, Ueno S, Shiraishi M, Okuda K, et al. Prognostic Impact of Preoperative Lymph Node Enlargement in Intrahepatic Cholangiocarcinoma: A Multi-Institutional Study by the Kyushu Study Group of Liver Surgery. Ann Surg Oncol. 2015; 22: 2269-2278.

39. Hu HJ, Mao H, Tan YQ, Shrestha A, Ma WJ, Yang Q, et al. Clinical value of preoperative serum CA 19-9 and CA 125 levels in predicting the resectability of hilar cholangiocarcinoma. Springer Plus. 2016; 5: 551.

40. Coelho R, Silva M, Rodrigues-Pinto E, Cardoso H, Lopes S, Pereira P, et al. CA 19-9 as a Marker of Survival and a Predictor of Metastization in Cholangiocarcinoma. GE Port J Gastroenterol. 2017; 24: 114-121.
Austin J Surg - Volume 6 Issue 4 - 2019

ISSN : 2381-9030 | www.austinpublishing group.com

Choi et al. @ All rights are reserved
Citation: Navarro JG, Rho SY, Lee JH, Choi GH, Han DH, Kim SK, et al. Prognostic Significance and Predictive Factors of Lymph Node Metastasis in Resectable Intrahepatic Cholangiocarcinoma. Austin J Surg. 2019; 6(4): 1168. 